\title{
Über den Zusammenhang der Temperatur und der Leistung eines Reaktors mit der Reaktivität und der Kühlung
}

\author{
Von H. Gaus und R. Schulten \\ Aus dem Max-Planck-Institut für Physik, Göttingen \\ (Z. Naturforschg. 9a, 964-968 [1954]; eingegangen am 11. September 1954)
}

\begin{abstract}
Aus der Diffusionsgleichung für die Neutronenvermehrung im Reaktor folgt, daß die Neutronendichte nur dann zeitlich konstant (stationärer Betrieb) ist, wenn die effektive Neutronenvermehrung genau gleich eins ist. Die Neutronenvermehrung nimmt mit steigender Temperatur ab. Beim stationären Betrieb stellt sich daher eine bestimmte, von der Kühlung im wesentlichen unabhängige Temperatur so ein, daß die effektive Neutronenvermehrung gleich eins wird. Die Reaktorleistung und die ihr proportionale Neutronendichte ist dann durch die Wärmemenge gegeben, die pro Sekunde aus dem Reaktor durch die Kühlung heraustransportiert wird. Diese Zusammenhänge werden mathematisch dargestellt.
\end{abstract}

Ü ber die Methoden zur Berechnung der kritischen Daten eines Reaktors liegt eine umfangreiche Literatur vor. In vielen Fällen wird sowohl für homogene als auch für heterogene Reaktoren die elementare Diffusionstheorie der Neutronen und die Fermische Age-Theorie angewandt, womit man für die kritischen Daten der Reaktoren mit der Erfahrung hinreichend übereinstimmende Ergebnisse erhält1,2. Man gewinnt jedoch noch keine Aussage über die Größe des Neutronenflusses bzw. die Reaktorleistung, was sich mathematisch darin äußert, daß die entsprechenden Gleichungen linear in $n$ (Neutronendichte) und homogen sind. In Wirklichkeit stellt sich aber beim Betrieb eines Reaktors eine ganz bestimmte Neutronendichte ein, die durch die in den oben erwähnten Gleichungen noch nicht berücksichtigte Temperaturabhängigkeit der Materialkonstanten bedingt ist. Die Berücksichtigung dieser Temperaturabhängigkeit ergibt nichtlineare Terme in den Diffusionsgleichungen. Dadurch wird die Größe der Neutronendichte festgelegt. Diese Rechnung soll im folgenden für den stationären Betrieb eines heterogenen Reaktors durchgeführt werden, bei dem sich zwischen den feststehenden Uranstäben und dem feststehenden Moderator ein Zwischenraum für das umfließende Kühlmittel befindet. Es ergeben sich dabei einfache Näherungsformeln für die Neutronendichte bzw. die Reaktorleistung, in Abhängigkeit von der Reaktivität und der Kühlung.

${ }^{1}$ W. Heisenberg, Theorie der Neutronen, Vorlesung Wintersemester 1950/51.

${ }^{2}$ S. Glasstone u. M. C. Edlund, Nuclear Reactor Theory, London.

\section{§ 1. Die Gleichungen für die Neutronendichte}

Die zeitliche Veränderung der thermischen Neutronendichte $n(\mathfrak{r}, t)$ ist durch

$$
\frac{\partial n}{\partial t}=D \operatorname{div}(v \operatorname{grad} n)-\Sigma_{\mathrm{a}} v n+k P \Sigma_{\mathrm{a}} v n
$$

bestimmt, wenn der Einfluß der verzögerten Neutronen vernachlässigt wird, was im stationären Falle streng richtig ist.

$\left[D=1 / 3 \lambda_{t}=\right.$ mittlere Diffusionskonstante der Neutronen im Reaktor [cm]; $v=$ mittlere Geschwindigkeit der thermischen Neutronen $[\mathrm{cm} / \mathrm{sec}] ; \Sigma_{\mathrm{a}}=$ mittlerer ,makroskopischer Absorptionsquerschnitt" $\left[\mathrm{cm}^{-1}\right]$; $P=$ Wahrscheinlichkeit, daß ein Neutron während des Abbremsprozesses im Reaktor verbleibt; $k=$ Vermehrungsfaktor für den unendlich großen Reaktor, gegeben durch die Zellenstruktur des Reaktors.]

In Gl. (1) stellt der erste Term rechts die Anzahl der Neutronen dar, die durch Diffusion auf Grund des Neutronenstromes $-D v \operatorname{grad} n$ pro sec und pro $\mathrm{cm}^{3}$ in das Einheitsvolumen hineinströmen. Der zweite gibt die mittlere Anzahl der pro sec und $\mathrm{cm}^{3}$ absorbierten Neutronen an. Der dritte Term stellt den Zuwachs der thermischen Neutronen dar, der durch das Abbremsen der schnellen Spaltungsneutronen auf thermische Geschwindigkeit entsteht. Diesen Zuwachs haben wir proportional zur Dichte der thermischen Neutronen gesetzt, was zulässig ist, sofern die Bremslänge der Spaltungsneutronen klein gegen die Reaktorabmessungen ist.

Die für die Lösung der obigen Gleichung erforderliche Randbedingung ist durch das Verschwinden der Dichte am Rande des Reaktorkernes gegeben. Die Wirkung des Reflektors wird berücksichtigt, indem man das Verschwinden der Dichte 
in einer bestimmten Entfernung $\delta$ außerhalb dieses Randes verlangt. Die Größe $\delta$ wird durch die Reflektortheorie bestimmt; für Graphit mit der Dichte 1,6 beträgt $\delta=53 \mathrm{~cm}$.

Man definiert einen Vermehrungsfaktor $k_{\text {eff }}$ für den endlichen Reaktor durch

$$
k_{\text {eff }}=k P /\left(1+B^{2} L^{2}\right)
$$

$\left[L=\sqrt{D / \Sigma_{\mathrm{a}}}=\right.$ Diffusionslänge der Neutronen; $B=$ geometrische Krümmung der Neutronendichte. Für einen zylinderförmigen Reaktor von der Länge $l$ und dem Radius $R$ ist z. B.:

$$
\begin{aligned}
B^{2} & =\beta_{r}{ }^{2}+\beta_{z}{ }^{2} \text { mit } \\
\beta_{r} & =2,405 /(R+\delta) ; \quad \beta_{z}=\pi /(l+2 \delta) .
\end{aligned}
$$

Hierbei ist $\delta$ die oben erwähnte, durch den Reflektor bedingte Verschiebung des Randes].

Drückt man in (1) $k$ durch $k_{\text {eff }}$ aus, so erhält man $\frac{1}{D} \frac{\partial n}{\partial t}=\operatorname{div}(v \operatorname{grad} n)$

$$
+\frac{1}{L^{2}}\left[-1+k_{\text {eff }}\left(1+B^{2} L^{2}\right)\right] v n .
$$

Eine Neutronenvermehrung ist nur für $k_{\text {eff }}>1$ möglich, wie man erkennt, wenn man

$$
\Delta n=-B^{2} n
$$

setzt, wobei $B^{2}$ durch die obigen Randbedingungen bestimmt ist. Hierbei ist $v$ als ortsunabhängig angenommen. Diese vorläufige Betrachtung ergibt eine stationäre Lösung

$$
n=A^{\prime \prime} J_{0}\left(\beta_{r} r\right) \cos \left(\beta_{z} z\right)
$$

nur für den Fall $k_{\text {eff }}=1$. Die Amplitude $A^{\prime \prime}$ bleibt unbestimmt.

\section{§ 2. Temperaturabhängigkeit, Wärmeübertragung und Übergang von der Neutronendichte zur Leistung}

Sämtliche oben eingeführten Materialkonstanten und die Geschwindigkeit der thermischen Neutronen $v$ sind temperaturabhängig. Erfahrungsgemäß macht sich die Temperaturabhängigkeit am stärksten beim Vermehrungsfaktor $k$ infolge der Vergrößerung der Resonanz-Einfangwahrscheinlichkeit bemerkbar ${ }^{3}$. Wir setzen:

$$
k_{\text {eff }}(T)=k_{\text {eff }}\left(T_{0}\right)-\Gamma\left(T-T_{0}\right),
$$

wobei $\Gamma \approx 1,5 \cdot 10^{-5}\left({ }^{0} \mathrm{C}\right)^{-1}$ ist $^{4}$. Diese Temperaturabhängigkeit bewirkt, daß sich auch für $k_{\text {eff }}\left(T_{0}\right)$ $>1$ ein stationärer Zustand einstellt, indem sich

${ }^{3}$ R. V. Moore et al., Inst. Electr. Engrs., Paper Jan. 1953. die Temperatur so weit erhöht, daß $k_{\text {eff }}(T)$ im Mittel gleich 1 wird. Wir definieren eine Temperatur $T^{*}$ durch

$$
\begin{gathered}
k_{\mathrm{eff}}\left(T^{*}\right)=1, \\
T^{*}=T_{0}+1 / \Gamma\left[k_{\mathrm{eff}}\left(T_{0}\right)-1\right] .
\end{gathered}
$$

Hiernach ist $T^{*}$ ein Maß für die Reaktivität des Reaktors. $T^{*}$ ist nach obigem ferner eine Art Mittelwert für die in Wirklichkeit ortsabhängige Temperatur $T$ beim stationären Betrieb des Reaktors.

Wählt man in (6) $T_{0}=T^{*}$ und setzt das temperaturabhängige $k_{\text {eff }}(T)$ in (4) für $k_{\text {eff }}$ ein, so erhält man für den stationären Fall

$$
\begin{aligned}
0= & \operatorname{div}(v \operatorname{grad} n) \\
& +\left[B^{2}+\frac{1}{L^{2}}\left(1+B^{2} L^{2}\right) \Gamma\left(T^{*}-T\right)\right] v n .
\end{aligned}
$$

Um die Verknüpfung der Temperatur mit der Kühlung darzustellen, ist es zweckmäßig, von der Neutronendichte zur Leistung überzugehen. Es sei z. B. in einem heterogenen Reaktor mit Uran in Stabform $Q(z)$ die Leistung pro $\mathrm{cm}$, die in einem bestimmten (z. B. dem in der Mitte des Reaktors parallel zur Zylinderachse gelegenen) Uranstab erzeugt wird. Sie ist proportional zur Neutronendichte $n^{\prime}$ im Uran, die wiederum proportional zur bisherigen Neutronendichte $n$ ist, die dem äquivalenten homogenen Reaktor entspricht. Es ist

$Q(z)=f \Sigma_{f} \cdot v \cdot n^{\prime} \cdot 180 \mathrm{MeV}$

$$
\text { (180 MeV = Spaltungsenergie), }
$$

worin $f$ der Querschnitt des Uranstabes und $\Sigma_{f}$ der makroskopische Absorptionsquerschnitt für Spaltung ist, für den man eine $1 / v$-Abhängigkeit annehmen darf. Der so entstehende Proportionalitätsfaktor ist also temperaturunabhängig, seine Größe ist für unsere weitere Rechnung belanglos.

Beim stationären Betrieb muß die durch Spaltung erzeugte Wärme durch die Oberfläche des Uranstabes abfließen und sich in einer Erwärmung des Kühlmittels wiederfinden. Mithin gilt:

$$
\begin{gathered}
Q(z)=\frac{F}{l} \alpha[T(z)-\vartheta(z)], \\
\frac{F}{l} \alpha[T(z)-\vartheta(z)] \mathrm{d} z=j c_{p} \mathrm{~d} \vartheta
\end{gathered}
$$

$[F=$ Oberfläche eines Uranstabes; $l=$ Länge eines Uranstabes; $F / l=$ Oberfläche pro $\mathrm{cm}$ Uranstab; $\alpha=$ Wärmeübertragungsfaktor $\left[\mathrm{cal} / \mathrm{cm}^{2} \sec { }^{0} \mathrm{C}\right] ; T(z)=$

${ }^{4}$ A. Weinberg, Diskussionsbemerkung, Proc. Kjeller Conf. Heavy Water Reactors, Kjeller 1953. 
Urantemperatur (an der Oberfläche des Stabes); $\vartheta(z)=$ Kühlmitteltemperatur; $c_{p}=$ spez. Wärme pro Gramm des Kühlmittels; $j=$ Kühlstrom [Gramm/sec] längs eines Uranstabes].

In (11) wurde die Wärmeleitung längs des Uranstabes vernachlässigt, was zulässig ist, zumal der Uranstab praktisch meistens unterteilt ist. Für den Wärmeübertragungsfaktor $\alpha$ gibt es empirische Formeln ${ }^{5,6}$. Er hängt von $j$, den geometrischen Abmessungen des Kühlkanals und den Materialkonstanten des Kühlmittels ab.

Aus (11) und (12) folgt

$$
\begin{aligned}
& T(z)=\frac{l}{F \alpha} Q(z)+\vartheta_{0}+\int_{-l / z}^{z} \frac{Q\left(z^{\prime}\right)}{j c_{p}} \mathrm{~d} z^{\prime}, \\
& \vartheta(z)=\vartheta_{0}+\int_{-l / z}^{z} \frac{Q\left(z^{\prime}\right) \mathrm{d} z^{\prime}}{j c_{p}} .
\end{aligned}
$$

Dabei ist $\vartheta_{0}$ die Eintrittstemperatur des Kühlmittels.

Wir betrachten im folgenden den technisch wichtigsten Fall, daß die Temperatur im Reaktor nur eine Funktion der Längsrichtung ( $z$-Koordinate) der Stäbe ist. Dies läßt sich nach (13), (14) bzw. (9) erreichen, wenn man $j c_{p}$ und $F \alpha$ in den verschiedenen Kanälen (verschiedene $r$-Koordinaten) proportional $J\left(\beta_{r} r\right)$, also proportional zur Radialabhängigkeit von Neutronendichte bzw. Leistung wählt.

Die Temperatur des Moderators ist im stationären Falle bei der von uns angenommenen Anordnung gleich der Temperatur des Kühlmittels, da die Wärmeerzeugung durch Abbremsung von Neutronen im Moderator praktisch keine Rolle spielt. Dementsprechend ist die mittlere thermische Geschwindigkeit der Neutronen durch $\vartheta$ festgelegt:

$$
v=v_{0} \sqrt{\vartheta^{\mathrm{abs}} / \vartheta_{0}^{\mathrm{abs}}}
$$

$\left[\vartheta^{\text {abs }}=\right.$ absolute Temperatur des Kühlmittels; $\vartheta_{0}$ abs $=$ absolute Eintrittstemperatur des Kühlmittels; $v_{0}=$ mittlere thermische Geschwindigkeit bei der Temperatur $\left.\vartheta_{0} \mathrm{abs}\right]$.

Da nach den obigen Bemerkungen $\vartheta$ nur eine Funktion von $z$ ist, können wir den ersten Term von Gl. (9) in den folgenden umformen

$$
\operatorname{div}(v \operatorname{grad} n)=v\left\{\Delta n+\frac{1}{v} \frac{\partial v}{\partial z} \frac{\partial n}{\partial z}\right\} .
$$

Dabei ist nach Gl. (15)

${ }^{5}$ Hütte I, Berlin 1949; J. D'Ans und E. Lax, Taschenbuch für Chemiker und Physiker, Berlin 1943.

$$
\frac{1}{v} \frac{\partial v}{\partial z}=\frac{1}{2 \vartheta^{\mathrm{abs}}} \cdot \frac{\mathrm{d} \vartheta}{\mathrm{d} z} .
$$

Wir können nun in (9) Zylinderkoordinaten einführen und die Radialabhängigkeit von $n$ durch Produktansatz

$$
n(r, z)=n(r) n(z)=J_{0}\left(\beta_{r} r\right) n(z)
$$

separieren. In der so erhaltenen Gleichung können wir statt $n(z)$ gemäß (10) die Leistungsdichte $Q(z)$ einführen. Wir erhalten

$$
\begin{aligned}
-\frac{\mathrm{d}^{2} Q}{\mathrm{~d} z^{2}} & +\frac{1}{2 \vartheta \mathrm{abs}} \cdot \frac{\mathrm{d} \vartheta}{\mathrm{d} z} \frac{\mathrm{d} Q}{\mathrm{~d} z} \\
& +\left[\beta_{z}{ }^{2}+\frac{1}{L^{2}}\left(1+B^{2} L^{2}\right) \Gamma\left(T^{*}-T\right)\right] Q=0 .
\end{aligned}
$$

Wir ersetzen hier im zweiten Term $\vartheta^{\text {abs }}(z)$ der Einfachheit halber durch einen konstanten Mittelwert $\overline{\vartheta^{\mathrm{abs}}}$ und setzen $T$ und $\mathrm{d} \vartheta / \mathrm{d} z$ nach (13), (14) ein. Es ergibt sich mit

$$
\begin{gathered}
x=\frac{z}{l^{\prime}}, l^{\prime}=l+2 \delta \quad[\operatorname{vgl} .(3)] \\
Q^{\prime \prime}+\pi^{2} Q+\frac{l^{\prime 2}}{L^{2}}\left(1+B^{2} L^{2}\right) \Gamma \cdot\left[\left(T^{*}-\vartheta_{0}\right)-\frac{l}{F \alpha} Q\right. \\
\left.-\frac{l^{\prime}}{j c_{p}} \int_{-l / 2 l^{\prime}}^{x} Q \mathrm{~d} x\right] Q+Q Q^{\prime} \frac{l^{\prime}}{2 \overline{\vartheta^{\mathrm{abs}} j c_{p}}}=0 .
\end{gathered}
$$

Man sieht die erwähnte Nichtlinearität der Temperaturterme in $Q$, durch die die Leistung auch ihrem Betrage nach festgelegt wird.

\section{§ 3. Bestimmung von Leistung und Temperatur}

Wir bestimmen die Leistung aus (20) zunächst unter der Annahme, daß die Form der Neutronendichte bzw. Leistungsdichte längs eines Stabes im wesentlichen cos-förmig ist. Wir dividieren (20) durch $Q$ und setzen dann

$$
Q(x)=A \cos (\pi x)
$$

in die linke Seite ein, quadrieren und integrieren von $-\frac{1}{2}$ bis $+\frac{1}{2}$. Das Ergebnis machen wir durch Variation von $A$ zum Minimum. Hieraus ergibt sich

$$
\begin{aligned}
A & =\frac{\pi}{l^{\prime}} \frac{j c_{p}\left(T^{*}-\vartheta_{0}\right)}{2 a+\sin \left(\frac{\pi}{2} \frac{l^{\prime}}{l}\right)+R} \\
\operatorname{mit} R & =\frac{\left(\frac{\pi^{2}}{2}-4\right) a^{2}+\frac{1}{2}\left[1+\frac{\pi^{2}}{2 \Gamma \overline{\vartheta^{\mathrm{abs}}}} \cdot \frac{L^{2}}{l^{2}\left(1+B^{2} L^{2}\right)}\right]^{2}}{2 a+\sin \left(\frac{\pi}{2} \frac{l}{l^{\prime}}\right)} .
\end{aligned}
$$

\footnotetext{
${ }^{6}$ H. Hausen, Wärmeübertragung im Gegenstrom,
} Gleichstrom und Kreuzstrom, Springer 1950. 
Hierbei ist

$$
a=\frac{j c_{p}}{F \alpha} \frac{l}{l^{\prime}},
$$

d. h. $a$ ist bis auf den Faktor $l / l^{\prime}$ gleich dem Quotienten aus der mittleren Temperaturdifferenz Uranoberfläche-Kühlmittel dividiert durch die Erwärmung des Kühlmittels, denn es muß nach (12)

$$
\frac{j c_{p}\left(\vartheta\left(\frac{l}{2}\right)-\vartheta\left(-\frac{l}{2}\right)\right)}{F \cdot \alpha \cdot(\overline{T-\vartheta})}=1
$$

sein. Wie früher bemerkt, kann $\alpha$ und folglich auch $a$ mit Hilfe empirischer Formeln berechnet werden. $k_{\text {eff }}$ und damit nach (8) $T^{*}$ kann aus den Reaktorabmessungen und Materialkonstanten bestimmt ${ }^{2,7}$ und durch Kontrollstäbe geregelt werden.

Durch Integration von $-l / 2$ bis $+l / 2$ ergibt sich die von einem Uranstab im ganzen abgegebene Leistung

$$
\bar{Q}=\frac{\pi}{2} \cdot l^{\prime} \cdot A \sin \left(\frac{\pi}{2} \frac{l}{l^{\prime}}\right) .
$$

Hiermit wird die Gesamtleistung unter den oben für die Radialabhängigkeit des Kühlstroms gemachten Voraussetzungen:

$$
Q_{\mathrm{ges}}=\bar{Q}_{\text {zentr }} N \cdot \frac{2(R+\delta)}{2,405 R} J_{1}\left(\frac{2,405 \cdot R}{R+\delta}\right) .
$$

Dabei bedeutet $N$ die Anzahl der Kanäle, $\bar{Q}_{\text {zentr. }}$ die Leistung des in der Mitte liegenden Uranstabes, und der letzte Faktor stellt eine Mittelung der nullten Bessel-Funktion über die Querschnittsfläche des Reaktors dar. Bei gegebenen Reaktorabmessungen und gegebenem $T^{*}$ ist die entnommene Leistung also ziemlich proportional zum Kühlstrom $j$; infolge der $j$-Abhängigkeit von $a$ ist der Leistungsanstieg etwas geringer als der von $j$.

Mit $Q(z)$ und den Gl. (13) und (14) können wir nun den Verlauf der Urantemperatur $T(z)$ und der Temperatur des Kühlmittels $\vartheta(z)$ bestimmen (siehe auch Gilliland ${ }^{8}$ ):

$$
\begin{array}{r}
T(z)=\frac{T^{*}-\vartheta_{0}}{2 a+\sin \left(\frac{\pi}{2} \frac{l}{l^{\prime}}\right)+R}\left\{\sin \left(\frac{\pi}{2} \frac{l}{l^{\prime}}\right)\right. \\
\left.+\pi a \cos \left(\frac{\pi z}{l^{\prime}}\right)+\sin \left(\frac{\pi z}{l^{\prime}}\right)\right\},
\end{array}
$$

$$
\begin{aligned}
\vartheta(z) & =\vartheta_{0} \\
& +\frac{T^{*}-\vartheta_{0}}{2 a+\sin \left(\frac{\pi}{2} \frac{l}{l^{\prime}}\right)+R}\left\{\sin \left(\frac{\pi}{2} \frac{l}{l^{\prime}}\right)+\sin \left(\frac{\pi z}{l^{\prime}}\right)\right\} .
\end{aligned}
$$

${ }^{7}$ E. A. Guggenheimer u. M. Pryce, Nucleonics 11, Nr. 2, p. 50 [1953].
Der Verlauf von $T(z)$ und $\vartheta(z)$ ist für ein typisches Beispiel eines luftgekühlten Reaktors in Abb. 1 aufgetragen.

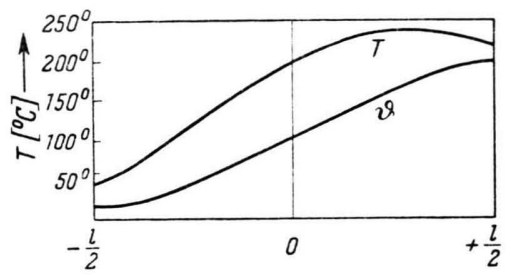

Abb. 1. Beispiel für Uran- und Kühlmitteltemperatur als Funktion des Ortes.

Der Maximalwert der Urantemperatur, der aus Materialgründen von besonderem Interesse ist, ergibt sich aus Gl. (27):

$T_{\max }=\left(T^{*}-\vartheta_{0}\right) \cdot \frac{\left\{\sqrt{ } 1+(\pi a)^{2}+\sin \left(\frac{\pi}{2} \frac{l}{l^{\prime}}\right)\right\}}{2 a+\sin \left(\frac{\pi}{2} \frac{l}{l^{\prime}}\right)+R}$.

Für die mittlere Temperatur erhält man:

$\bar{T}=\left(T^{*}-\vartheta_{0}\right) \cdot \frac{2 a+\frac{l}{l^{\prime}}}{2 a+\sin \left(\frac{\pi}{2} \frac{l}{l^{\prime}}\right)+R} \cdot \frac{\sin \left(\frac{\pi}{2} \frac{l}{l^{\prime}}\right)}{\frac{l}{l^{\prime}}}$.

Man sieht, daß bei gegebenem $T^{*}$ die Urantemperaturen $T_{\max }$ und $\bar{T}$ von $a$ praktisch wenig abhängen, d. h. ziemlich unabhängig von der Art der Kühlung und der entnommenen Leistung sind. Dagegen ist die durch Integration von (28) folgende Erwärmung des Kühlmittels

$$
\vartheta\left(\frac{l}{2}\right)-\vartheta_{0}=\left(T^{*}-\vartheta_{0}\right) \cdot \frac{2 \sin \frac{\pi l}{2 l^{\prime}}}{2 a+\sin \frac{\pi l}{2 l^{\prime}}+R}
$$

wesentlich von $a$ abhängig und kann auch bei gegebener Leistung $\bar{Q}$ bzw. $Q$ durch Wahl des Strömungsquerschnittes des Kühlmittels noch wesentlich variiert werden.

Es sei noch einmal darauf hingewiesen, daß unsere Ergebnisse (21) bis (31) nur für den stationären Betrieb gelten. Die Größe des Neutronenflusses läßt sich aus der Leistung mit Hilfe von Gl. (10) bestimmen. Der in $R$ enthaltene Mittelwert $\overline{\vartheta^{\mathrm{abs}}}$, der sich eigentlich erst aus (31) ergibt, kann bei der Rechnung zunächst genähert eingesetzt werden. Man findet einen Näherungswert, indem man statt des Ausdruckes (22) für $A$ den Wert $A^{\prime}$ nach (36) einsetzt.

${ }^{8}$ E. R. Gilliland et al. in The Science and Engineering of Nuclear Power, Addison-Wesley-Press 1949. 


\section{§ 4. Einfluß der Temperatureffekte auf die Form der Neutronendichte}

Um den Einfluß der Temperatureffekte auf die (im vorigen Paragraphen angenommene) cos-Form der Neutronendichte bzw. Leistung zu erkennen, wollen wir die Gl. (20) noch durch eine Art Störungsrechnung näherungsweise lösen. Für hinreichend kleine Amplituden $A$ ist der Einfluß der in $Q$ quadratischen Terme in (20) gering gegen die in $Q$ linearen Terme. Wir setzen deshalb in diesen Termen die ,,ungestörte Lösung “

$$
Q=A^{\prime} \cos (\pi x)
$$

ein, wodurch wir eine Gleichung der Form

$$
Q^{\prime \prime}+\pi^{2} Q+f(x)=0
$$

erhalten. (Wir haben hier den ersten Term der eckigen Klammer von (20) mit zu den ,,Störgliedern" genommen.) Gl. (33) läßt sich integrieren mit dem Ansatz

$$
\begin{aligned}
Q=A^{\prime} \cos \pi x+C & +B \cos 2 \pi x+D \sin 2 \pi x \\
& +E x \sin \pi x+F \sin \pi x,
\end{aligned}
$$

wobei sich ergibt

$$
\begin{aligned}
& C=\left(\frac{A^{\prime}}{\pi}\right)^{2} \frac{1}{2} \frac{l^{\prime 2}}{L^{2}}\left(1+B^{2} L^{2}\right) \frac{\Gamma l}{F \alpha}, \\
& B=-\left(\frac{A^{\prime}}{\pi}\right)^{2} \frac{1}{6} \frac{l^{\prime 2}}{L^{2}}\left(1+B^{2} L^{2}\right) \frac{\Gamma l}{F \alpha},
\end{aligned}
$$

$$
\begin{gathered}
D=-\left(\frac{A^{\prime}}{\pi}\right)^{2}\left(\frac{1}{6 \pi} \frac{l^{\prime 2}}{L^{2}}\left(1+B^{2} L^{2}\right) \frac{\Gamma l^{\prime}}{j c_{p}}\right. \\
\left.+\frac{1}{12} \frac{l^{\prime}}{2 \overline{\vartheta^{\mathrm{abs}}} j c_{p}}\right) \\
E=\frac{A^{\prime}}{2 \pi} \frac{l^{\prime 2}}{L^{2}}\left(1+B^{2} L^{2}\right) \Gamma\left(T^{*}-\vartheta_{0}\right) \\
+\left(\frac{A}{\pi}\right)^{2} \frac{l^{\prime 2}}{L^{2}}\left(1+B^{2} L^{2}\right) \frac{\Gamma l^{\prime}}{j c_{p}} \frac{\sin \left(\frac{\pi}{2} \frac{l}{l^{\prime}}\right)}{2}
\end{gathered}
$$

Die noch unbestimmten Koeffizienten $A^{\prime}$ und $F$ folgen aus den beiden Randbedingungen (Verschwinden der Neutronendichte an den Stellen $\left.x= \pm-^{-}-\right)$:

$$
F=0, A^{\prime}=\frac{\pi}{l^{\prime}} \frac{j c_{p}\left(T^{*}-\vartheta_{0}\right)}{\frac{8}{3} a+\sin \frac{\pi l}{2 l^{\prime}}} .
$$

Diese Lösung ist jedenfalls so lange brauchbar, wie die Koeffizienten (35) klein gegen $A^{\prime}$ sind. Das ist bei Graphit-Reaktoren einiger Megawatt Leistung der Fall, wenn die Erwärmung des Kühlgases nicht $\mathrm{zu}$ groß ist. Bei $\mathrm{D}_{2} \mathrm{O}$-Reaktoren ist der Anwendungsbereich von (35) noch günstiger, da für diese $l^{\prime} / L$ etwa fünfmal kleiner als für Graphitreaktoren ist. Allerdings muß bei $\mathrm{D}_{2} \mathrm{O}$-Reaktoren der Einfluß des Reflektors genauer als in unserer Rechnung [z. B. Gl. (3)] berücksichtigt werden.

Herrn Prof. Wirtz möchten wir für verschiedene Unterhaltungen vielmals danken.

\title{
Experimenteller Beitrag zur Theorie des äußeren lichtelektrischen Effektes kompakter Alkalimetalle
}

\author{
Von R. SuHRManN \\ Aus dem Institut für Physikalische Chemie und Elektrochemie \\ der Technischen Hochschule Braunschweig \\ (Z. Naturforschg. 9a, 968-973 [1954]; eingegangen am 27. September 1954)
}

\begin{abstract}
Die spektrale Empfindlichkeitskurve sehr reiner Oberflächen kompakten Kaliums besitzt bei $400 \mathrm{~m} \mu$ ein Maximum, wenn die Empfindlichkeit auf die von der Kathode absorbierte (,einfallende“) Strahlung bezogen wird. Von der langwelligen Grenze bis zum Maximum läßt sich die Kurve mittels der Fowlerschen Theorie des ,,Oberflächeneffektes" darstellen. - Wegen der starken Abnahme des optischen Absorptionskoeffizienten dringt die Strahlung nach kurzen Wellen hin in zunehmendem Maße tiefer in das Metall ein, als der ,Austrittstiefe“ der Photoelektronen entspricht. Dies ist der Grund für den Abfall der Empfindlichkeitskurve nach dem Überschreiten des Maximums. Berechnet man die spektrale Photoelektronenausbeute innerhalb der Austrittstiefe (,,wahre“" Empfindlichkeit), so verschwindet das Maximum. Die Ausbeute steigt nunmehr im Anschluß an die langwellige Grenze bis etwa $400 \mathrm{~m} \mu$ steil an, um unterhalb dieser Wellenlänge nur noch schwach zuzunehmen. Das spektrale Maximum der üblichen Empfindlichkeitskurve ist also auf die Wellenlängen-Abhängigkeit des Absorptionskoeffizienten und die geringe Austrittstiefe der Photoelektronen zurückzuführen.
\end{abstract}

